LA-UR-95-3393, LBL-37786

\title{
Dissipation and Decoherence in Mean Field Theory
}

\author{
Salman Habib ${ }^{1}$, Yuval Kluger ${ }^{2}$, Emil Mottola ${ }^{1}$, and Juan Pablo Paz ${ }^{3}$ \\ ${ }^{1}$ Theoretical Division \\ Los Alamos National Laboratory \\ Los Alamos, NM 87545, USA \\ ${ }^{2}$ Nuclear Science Division, MS 70A-3307 \\ Lawrence Berkeley National Laboratory \\ Berkeley, CA 94720, USA \\ ${ }^{3}$ Departamento de Fisica, FCEN, UBA \\ Pabellon 1, Ciudad Universitaria \\ 1428 Buenos Aires, Argentina
}

\begin{abstract}
The time evolution of a closed system of mean fields and fluctuations is Hamiltonian, with the canonical variables parameterizing the general time-dependent Gaussian density matrix of the system. Yet, the evolution manifests both quantum decoherence and apparent irreversibility of energy flow from the coherent mean fields to fluctuating quantum modes. Using scalar QED as an example we show how this collisionless damping and decoherence may be understood as the result of dephasing of the rapidly varying fluctuations and particle production in the time varying mean field.
\end{abstract}


Mean field methods have a long history in such diverse areas as atomic physics (Born-Oppenheimer), nuclear physics (Hartree-Fock), condensed matter (BCS) and statistical physics (Landau-Ginzburg), quantum optics (coherent/squeezed states), and semiclassical gravity. Because no higher than second moments of the fluctuations are incorporated, the mean field approximation is related to a Gaussian variational ansatz for the wave function of the system. The broad applicability of the approximation, as well as the variety of different approaches to it in the literature makes it worthwhile to exhibit its general features unobscured by the particulars of specific applications. Accordingly, our first purpose in this Letter is to demonstrate the equivalence of the time-dependent mean field approximation to the general Gaussian ansatz for the mixed state density matrix $\rho$, and to underline its Hamiltonian structure.

The Hamiltonian nature of the evolution makes it clear from the outset that the mean field approximation does not introduce dissipation or time irreversibility at a fundamental level. Nevertheless, typical evolutions seemingly manifest an arrow of time, in the sense that energy flows from the mean field to the fluctuations without returning over times of physical interest [Fig. 1]. Closely connected to this effective dissipation is the phenomenon of quantum decoherence [1], i.e., the suppression with time of the overlap between wave functions corresponding to two different mean field evolutions [Fig. 2]. This decoherence is the reason why quantum superpositions of different mean field states are difficult to observe in nature, and is crucial to understanding the quantum to classical transition in macroscopic systems. Our second aim in this Letter is to present an explicit example of a quantum field theory (scalar QED) treated in mean field approximation where these effects are observed, and to provide a clear physical explanation of the behavior in terms of dephasing of the fluctuations, i.e., the averaging to zero of their rapidly varying phases on time scales short compared to the collective motion of the mean field(s).

To expose the general structure of the time-dependent mean field (or Gaussian) approximation consider first a one-dimensional harmonic oscillator with Hamiltonian,

$$
H_{o s c}(q, p ; t)=\frac{1}{2}\left(p^{2}+\omega^{2}(t) q^{2}\right)
$$

where the frequency $\omega(t)$ is a smooth function of time, otherwise unspecified for the moment. The most general Gaussian ansatz for the mixed state normalized density matrix may be presented as

$$
\begin{aligned}
\left\langle x^{\prime}|\rho| x\right\rangle= & \left(2 \pi \xi^{2}\right)^{-\frac{1}{2}} \exp \left\{i \frac{\bar{p}}{\hbar}\left(x^{\prime}-x\right)-\frac{\mu^{2}+1}{8 \xi^{2}}\left[\left(x^{\prime}-\bar{q}\right)^{2}+(x-\bar{q})^{2}\right]\right. \\
& \left.+i \frac{\eta}{2 \hbar \xi}\left[\left(x^{\prime}-\bar{q}\right)^{2}-(x-\bar{q})^{2}\right]+\frac{\mu^{2}-1}{4 \xi^{2}}\left(x^{\prime}-\bar{q}\right)(x-\bar{q})\right\},
\end{aligned}
$$


in the coordinate representation. The five parameters $(\bar{q}, \bar{p}, \xi, \eta, \mu)$ of this Gaussian may be identified with the two mean values, $\bar{q}=\langle q\rangle \equiv \operatorname{Tr}(q \rho), \bar{p}=\langle p\rangle \equiv \operatorname{Tr}(p \rho)$, and the three symmetrized variances via

$$
\begin{gathered}
\left\langle(q-\bar{q})^{2}\right\rangle=\xi^{2}, \quad\langle(p q+q p-2 \bar{q} \bar{p})\rangle=2 \xi \eta, \\
\left\langle(p-\bar{p})^{2}\right\rangle=\eta^{2}+\frac{\hbar^{2} \mu^{2}}{4 \xi^{2}} .
\end{gathered}
$$

The one anti-symmetrized variance is fixed by the commutation relation, $[q, p]=i \hbar$. The parameter $\mu$ measures the degree to which the state is mixed: $\operatorname{Tr} \rho^{2}=\mu^{-1} \leq 1$, the equality holding for pure states. If the state is pure, $\rho=|\psi\rangle\langle\psi|$, and only two of the three symmetrized variances in (3) are independent.

The Gaussian ansatz for the density matrix is preserved under time evolution. In the Schrödinger picture $\rho$ evolves according to the Liouville equation, $\dot{\rho}=-i[H, \rho]$. Substitution of the Gaussian form (2) into this equation with Hamiltonian (11) and equating coefficients of $x, x^{\prime}, x^{2}, x^{\prime 2}$ and $x x^{\prime}$ gives five evolution equations for the five parameters specifying the Gaussian,

$$
\begin{gathered}
\dot{\bar{q}}=\bar{p} ; \quad \dot{\bar{p}}=-\omega^{2}(t) \bar{q} \\
\dot{\xi}=\eta ; \quad \dot{\eta}=-\omega^{2}(t) \xi+\frac{\hbar^{2} \mu^{2}}{4 \xi^{3}}
\end{gathered}
$$

and $\dot{\mu}=0$. Since $\mu$ is a constant and the von Neumann entropy, $-\operatorname{Tr} \rho \ln \rho$ of the state (2) is a (monotonic) function of $\mu$ alone, this quantity is also a constant of the motion. Evolution equations for the diverse applications of the time-dependent mean field approximation reduce to (multiple copies of) equations of precisely the general form of (仼), with $\omega^{2}(t)$ a different self-consistently determined function of the coordinates and time, depending on the application. This establishes the equivalence between mean field methods and Gaussian density matrices for all evolutions of the form of Eqns. (4). We give explicit examples below.

An essential property of the evolution equations (4) is that they are Hamilton's equations (hence, time reversible) for an effective classical Hamiltonian [2], with $\eta$ playing the role of the momentum conjugate to $\xi$,

$$
H_{e f f}(\bar{q}, \bar{p} ; \xi, \eta)=\operatorname{Tr}(\rho H)=\frac{1}{2}\left(\bar{p}^{2}+\eta^{2}\right)+V_{e f f}
$$

and $V_{\text {eff }}(\bar{q}, \xi)$ depending on the particular form of $\omega^{2}(\bar{q}(t), \xi(t) ; t)$. For example, if the original system is an anharmonic double well with quantum Hamiltonian

$$
H(q, p)=\frac{1}{2} p^{2}+\frac{\lambda}{4}\left(q^{2}-v^{2}\right)^{2}
$$

then the (large N) mean field equations of motion are identical to Eqs. (4) with $\omega^{2}(t)=\lambda\left(\bar{q}^{2}(t)+\xi^{2}(t)-v^{2}\right)$. In this case,

$$
V_{e f f}(\bar{q}, \xi)=\frac{\lambda}{4}\left(\bar{q}^{2}+\xi^{2}-v^{2}\right)^{2}+\frac{\hbar^{2} \mu^{2}}{8 \xi^{2}} .
$$


The resulting evolution equations (14) are now quite non-linear (and chaotic). The last "centrifugal" term in the effective potential is a manifestation of the quantum uncertainty principle which prevents the Gaussian width $\xi$ from shrinking to zero.

The unitary operator $U(t)$ which effects the time evolution of the density matrix (目),

$$
\rho(t)=U(t) \rho(0) U^{\dagger}(t), \quad U(t)=\exp \left(-i \int_{0}^{t} H d t\right)
$$

is given explicitly in the coordinate basis by

$$
\left\langle x^{\prime}|U(t)| x\right\rangle=(2 \pi i \hbar v(t))^{-\frac{1}{2}} \exp \left\{\frac{i}{2 \hbar v(t)}\left(u(t) x^{2}+\dot{v}(t) x^{\prime 2}-2 x x^{\prime}\right)\right\}
$$

in terms of the two linearly independent solutions to the classical evolution equation,

$$
\left(\frac{d^{2}}{d t^{2}}+\omega^{2}(t)\right)\left(\begin{array}{c}
u \\
v
\end{array}\right)=0 ; \quad \begin{aligned}
& u(0)=\dot{v}(0)=1 \\
& \dot{u}(0)=v(0)=0
\end{aligned} .
$$

The Gaussian dynamics may be expressed as well by means of a Fock representation of the time dependent Heisenberg operators,

$$
\begin{aligned}
& q(t)=U^{\dagger}(t) q(0) U(t)=\bar{q}(t)+a f(t)+a^{\dagger} f^{*}(t) \\
& p(t)=U^{\dagger}(t) p(0) U(t)=\bar{p}(t)+a \dot{f}(t)+a^{\dagger} \dot{f}^{*}(t)
\end{aligned}
$$

where $\left[a, a^{\dagger}\right]=1$. The complex mode functions $f$ satisfy the evolution equation (10) and the Wronskian condition,

$$
f \dot{f}^{*}-\dot{f} f^{*}=i \hbar
$$

showing that Gaussian time evolution is essentially classical, with $\hbar$ appearing only in the time independent condition (12) enforcing the quantum uncertainty relation. There is considerable latitude to redefine $f$ by the Bogoliubov transformation $f \rightarrow$ $\cosh \gamma e^{i(\theta+\phi)} f+\sinh \gamma e^{i(\theta-\phi)} f^{*}$ without affecting the Wronskian condition (12). Such Bogoliubov transformations form a noncompact Lie group, the metaplectic group $M p(2)$, which is a double covering of the symplectic group of classical Hamiltonian dynamics, $S p(2) \cong S U(1,1)$, whose Lie algebra is generated by the three symmetric bilinears $a a, a^{\dagger} a^{\dagger}$, and $a a^{\dagger}+a^{\dagger} a$. If the $a$ and $a^{\dagger}$ operators are appended to these three, the algebra again closes upon itself, forming a five parameter group $I M p(2)$ [3]. The unitary evolution (8), (9) of the Gaussian density matrix (2) is an explicit representation of this group.

The group structure can be exploited to choose a basis in which all expectation values vanish, except

$$
\left\langle a^{\dagger} a\right\rangle=\left\langle a a^{\dagger}\right\rangle-1 \equiv N \geq 0
$$


The Gaussian density matrix is diagonal in the corresponding $a^{\dagger} a$ time-independent number basis,

$$
\left\langle n^{\prime}|\rho| n\right\rangle=\frac{2 \delta_{n^{\prime} n}}{\mu+1}\left(\frac{\mu-1}{\mu+1}\right)^{n},
$$

with $\mu=2 N+1$ and $\xi^{2}(t)=\mu|f(t)|^{2}$. Upon identifying $\mu=\operatorname{coth}(\hbar \omega / 2 k T)$, the diagonal form (14) will be recognized as a thermal density matrix at temperature $T$. The pure state Gaussian wave function $(\mu=1)$ corresponds therefore to a coherent, squeezed zero temperature vacuum state. The smoothness of the finite temperature classical limit $\hbar \mu \rightarrow 2 k T / \omega$ as $\hbar \rightarrow 0, \mu \rightarrow \infty$ shows that quantum and thermal fluctuations are treated by the mean field approximation in a unified way.

By making another $I M p(2)$ group transformation it is always possible to diagonalize (11) at any given time, bringing the quadratic Hamiltonian into the standard harmonic oscillator form, $H_{\text {osc }}=\frac{\hbar \omega}{2}\left(\tilde{a} \tilde{a}^{\dagger}+\tilde{a}^{\dagger} \tilde{a}\right)$ with $\tilde{a}$ time dependent. This time dependent basis is defined by the relations,

$$
\begin{aligned}
& q(t)=\tilde{a} \tilde{f}+\tilde{a}^{\dagger} \tilde{f}^{*} \quad p(t)=-i \omega \tilde{a} \tilde{f}+i \omega \tilde{a}^{\dagger} \tilde{f}^{*} \\
& \tilde{f}(t)=\sqrt{\frac{\hbar}{2 \omega(t)}} \exp \left(-i \int_{0}^{t} d t^{\prime} \omega\left(t^{\prime}\right)\right),
\end{aligned}
$$

in place of (11). In the $\tilde{a}^{\dagger} \tilde{a}$ number basis, $\rho$ is no longer diagonal, $\langle\tilde{a}\rangle,\langle\tilde{a} \tilde{a}\rangle$, etc., are non-vanishing, and $\tilde{N} \equiv\left\langle\tilde{a}^{\dagger} \tilde{a}\right\rangle \neq N$ in general, becoming equal only in the static case of constant $\omega$.

If $\omega(t)$ varies slowly in time, an adiabatic invariant may be constructed from the Hamilton-Jacobi equation corresponding to the effective classical Hamiltonian (5). By a simple quadrature we find the adiabatic invariant,

$$
\frac{W}{2 \pi \hbar}=\frac{\langle H\rangle}{\hbar \omega}-\frac{\mu}{2}=\tilde{N}-N
$$

Since $N$ is time independent, $\tilde{N}(t)$ is an adiabatic invariant of the evolution. On the other hand, the phase angle conjugate to the action variable $W$ varies rapidly in time. Since the diagonal matrix elements of $\rho$ in the $\tilde{N}$ basis are independent of this phase angle, they are slowly varying, whereas the off-diagonal matrix elements of $\rho$ in this basis (which depend on the phase angle) are rapidly varying functions of time. If we are interested only in the effects of the fluctuations on the more slowly varying mean fields it is natural to define an effective density matrix $\rho_{e f f}(t)$ by time-averaging the density matrix (2), thereby truncating $\rho$ to its diagonal elements only, in the adiabatic $\tilde{N}$ basis 四. Clearly, for this truncation to be justified there must be very efficient phase cancellation, i.e. dephasing, either by averaging the fluctuations over time or by summing over many independent fluctuating degrees of freedom at a fixed time. 
Obtaining the general form of the diagonal matrix elements of $\rho$ in the $\tilde{N}$ basis is straightforward but the result is rather unwieldy. Here we restrict our attention to the case of a pure state with vanishing $\bar{q}$ mean field. Using the methods of Ref. [5], one finds simply

$$
\left.\langle\tilde{n}=2 \ell|\rho| \tilde{n}=2 \ell\rangle\right|_{\substack{\mu=1 \\ \bar{q}=\bar{p}=0}}=\frac{(2 \ell-1) ! !}{2^{\ell} \ell !} \operatorname{sech} \gamma \tanh ^{2 \ell} \gamma
$$

with $\rho_{\tilde{n} \tilde{n}}=0$ for $\tilde{n}$ odd and $\gamma(t)$ the parameter of the Bogoliubov transformation between the $a$ and $\tilde{a}$ bases. It is given explicitly by $\sinh ^{2} \gamma=\tilde{N}=|\dot{f}+i \omega f|^{2} / 2 \hbar \omega$.

Decoherence is also addressable within the same framework. Consider the case where $\omega(t)$ is a function of one external mean field degree of freedom $A(t)$. If only the evolution of $A$ is of interest, then the fluctuating modes described by $f(t)$ may be treated as the "environment." To solve for the evolution of the reduced density matrix of $A$, one needs to compute the influence functional. This is a functional of two trajectories $A_{1}(t)$ and $A_{2}(t)$ (corresponding to two different evolution operators $U_{1}(t)$ and $\left.U_{2}(t)\right)$, and is defined by

$$
F_{12}(t) \equiv \exp \left(i \Gamma_{12}(t)\right) \equiv \operatorname{Tr}\left(U_{1}(t) \rho(0) U_{2}^{\dagger}(t)\right)
$$

Explicit evaluation may be carried out using (91). Restricting again to pure states with vanishing $\bar{q}$ mean fields we find

$$
\left.\Gamma_{12}\right|_{\substack{\mu=1 \\ \bar{q}=\bar{p}=0}}=-\frac{i}{2} \ln \left\{\frac{i \hbar}{\left|f_{1} f_{2}\right|}\left(\frac{f_{1} f_{2}^{*}}{f_{1} \dot{f}_{2}^{*}-\dot{f}_{1} f_{2}^{*}}\right)\right\}
$$

in terms of the two sets of mode functions $f_{1}(t)$ and $f_{2}(t)$ which satisfy (10) and (12). This $\Gamma_{12}$ is precisely the closed time path (CTP) effective action functional which generates the connected real time $n$-point vertices in the quantum theory [6]. For a pure initial state, the absolute value of $F_{12}$ measures the overlap of the two different evolutions at some time $t$, beginning with the same initial $|\psi(0)\rangle$. In mean field theory, instead of evaluating $\Gamma_{12}$ for two arbitrary trajectories, the evaluation is over trajectories determined by the self-consistent evolution of the closed system, beginning with two different initial mean fields.

For an explicit field theoretic example consider scalar QED with no scalar selfcoupling. In the large $N$ limit, the evolution of electric fields and charged matter field fluctuations may be described in the self-consistent mean field or Gaussian approximation [6]. For a spatially homogeneous electric field in the $z$ direction, in the gauge $\vec{A}=A(t) \hat{z}$, the time evolution equations read

$$
\ddot{A}(t)=\left\langle j_{z}(t)\right\rangle=\frac{2 e}{V} \sum_{\vec{k}}\left(k_{z}-e A(t)\right) \quad\left|f_{\vec{k}}(t)\right|^{2}(2 N(\vec{k})+1)
$$




$$
\left[\frac{d^{2}}{d t^{2}}+(\vec{k}-e \vec{A}(t))^{2}+m^{2}\right] f_{\vec{k}}(t) \quad=0
$$

with the Wronskian condition (12) holding for every discrete wave number $\vec{k}$ in the finite volume $V$. In field theory there are an infinite number of quantum fluctuating modes $f_{\vec{k}}$ of the charged scalar field, each varying rapidly in time with its own characteristic frequency, $\omega_{\vec{k}}^{2}(t)=(\vec{k}-e \vec{A}(t))^{2}+m^{2}$. The Gaussian density matrix is an infinite product of Gaussians each of the form (2) and there are an infinite number of Bogoliubov parameters $\gamma(\vec{k} ; t)$, one for each $\vec{k}$. The equations of motion (20) are again Hamiltonian in structure. In this case the effective classical Hamiltonian,

$$
H_{e f f}=\frac{E^{2}}{2}+\frac{1}{V} \sum_{\vec{k}}\left[\left|\dot{f}_{\vec{k}}\right|^{2}+\omega_{\vec{k}}^{2}\left|f_{\vec{k}}\right|^{2}\right](2 N(\vec{k})+1)
$$

describes charged particle production in the electric field $E=-\dot{A}$ by the Schwinger mechanism and the effects of the current $\left\langle j_{z}(t)\right\rangle$ generated by these charged particles back on the electric field, through the semiclassical Maxwell equation in (20). The mean value of the scalar field itself is zero so that we may use the expressions (17) and (19) for the effective density matrix and decoherence functional of the charged field fluctuations. The values, $\rho_{2 \ell}$ given by (17) are then the probabilities of observing $\ell$ charged particle pairs in the adiabatic $\tilde{N}$ basis. The diagonal matrix elements of $\rho$ for odd $\tilde{n}$ vanish because particles can only be created in pairs from the vacuum.

In this specific model we present numerical results (in $1+1$ dimensions with vacuum initial conditions) on damping and decoherence of the mean electric field in Figs. 1 and 2. The plasma oscillations of the mean electric field on timescales long compared to $\omega_{\vec{k}}^{-1}$ are clearly seen, as well as the apparently irreversible flow of energy from the electric field towards the charged particle modes. In Fig. 1 we plot both the Boltzmann entropy and the von Neumann entropy of the effective density matrix, for comparison. These are defined by

$$
\begin{aligned}
S_{B} & \equiv \sum_{\vec{k}}\{(\tilde{N}(\vec{k})+1) \ln (\tilde{N}(\vec{k})+1)-\tilde{N}(\vec{k}) \ln \tilde{N}(\vec{k})\} \\
S_{\text {eff }} & \equiv-\operatorname{Tr} \rho_{\text {eff }} \ln \rho_{e f f}=-\sum_{\vec{k}} \sum_{\ell=0}^{\infty} \rho_{2 \ell}(\vec{k}) \ln \rho_{2 \ell}(\vec{k})
\end{aligned}
$$

respectively. Both display general increase during intervals of particle creation [7], when the electric field is sufficiently strong for the Schwinger pair creation mechanism to be effective. Neither quantity is a strictly monotonic function of time (no $H$ theorem). Since the charged particle modes $f_{\vec{k}}$ interact with the mean electric field but not directly with each other, the effective damping observed is certainly collisionless, and may be understood as due to dephasing similar to that responsible for Landau 

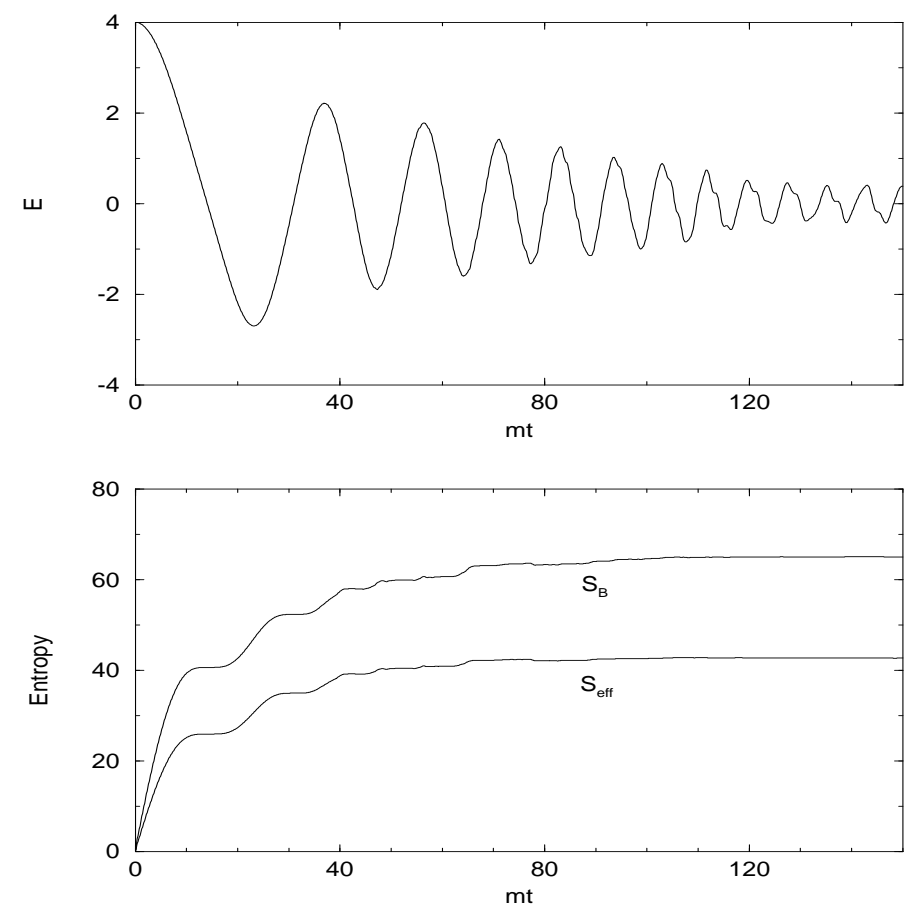

Figure 1: Evolution of the electric field, and the Boltzmann and effective entropies. The electric field is expressed in units of $E_{c}=m^{2} c^{3} / e \hbar$. Pair creation is rapid when $|E|>E_{c}$.
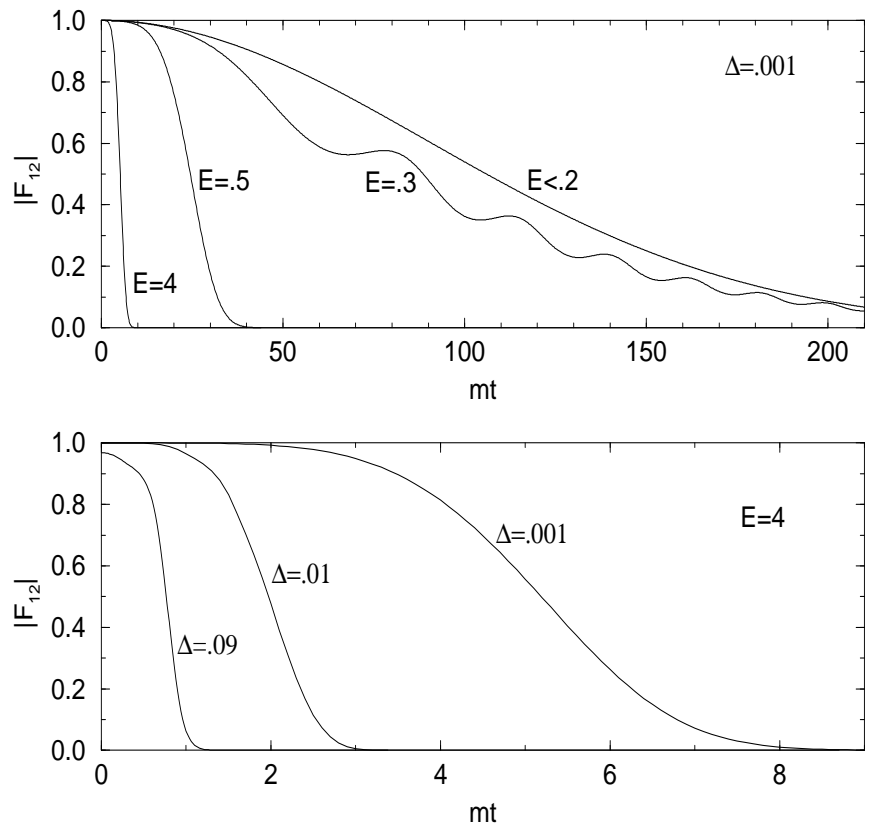

Figure 2: Absolute value of the decoherence functional as a function of time. The two field values are $E$ and $E-\Delta$. The top figure shows (for fixed $\Delta$ ) the sharp dependence of decoherence on particle production when $|E| \geq .2 E_{c}$. The second illustrates the relatively milder dependence on $\Delta$. 
damping of collective modes in classical electromagnetic plasmas. The entropy $S_{\text {eff }}$ of the effective density matrix provides a precise measure of the information lost by treating the phases as random. The Boltzmann "entropy" would be expected to equal $S_{\text {eff }}$ only in true thermodynamic equilibrium, which is not achieved in the collisionless approximation of Eqns. (20). Otherwise we see from Fig. 1 that $S_{B}$ generally overestimates the amount of information lost by phase averaging.

That decoherence is closely related to the same dephasing of the particle modes is seen most clearly by comparing the absolute value of $F_{12}$ for different initial electric fields. Decoherence is very slow for electric fields less than the Schwinger pair production threshold but becomes very rapid above it [8]. This shows the strong dependence of the decoherence process on the same particle production by the mean field.

The authors wish to acknowledge several helpful discussions with F. Cooper and A. Kovner.

\section{References}

[1] See e.g., Physical Origins of Time Asymmetry, J. J. Halliwell, J. Pérez-Mercader, and W. H. Zurek, eds. (Cambridge Univ. Press, Cambridge, 1994), and references therein.

[2] A. K. Rajagopal and J. T. Marshall, Phys. Rev. A 26, 2977 (1982), where the pure state case $\mu=1$ only is considered.

[3] R. G. Littlejohn, Phys. Rep. 138, 193 (1986).

[4] B. L. Hu and D. Pavon, Phys. Lett. B180, 329 (1986);

H. E. Kandrup, Phys. Rev. D 37, 3305 (1988); ibid 38, 1773 (1988).

[5] L. S. Brown and S. J. Carson, Phys. Rev. A 20, 2486 (1979).

[6] F. Cooper, S. Habib, Y. Kluger, E. Mottola, J. P. Paz, and P. R. Anderson, Phys. Rev. D 50, 2848 (1994), and references therein.

[7] E. Calzetta and B. L. Hu, Phys. Rev. D 40, 656 (1989).

[8] C. Kiefer, Phys. Rev. D 46, 1658 (1992). 